\title{
Rare Case of Duplication Cyst was Misdiagnosed as Pancreatic Pseudocyst
}

\author{
Imad Absah ${ }^{\mathrm{a}, \mathrm{c}}$, Deborah K. Freese ${ }^{\mathrm{b}}$
}

\begin{abstract}
A 7-year-old boy presents with a history of recurrent pancreatitis. His abdominal pain started in 2007, but his first episode of pancreatitis was in January 2010. Since that time has had at least five known episodes of pancreatitis with mild elevation of his amylase and lipase (range: 200 - 300). There is no history of abdominal trauma and he had negative genetic testing for the hereditary pancreatitis (CFTR, PRSS1, and SPINK1 gene mutations). He had numerous imaging studies revealing a persistent cystic lesion near the pancreatic head, since his first episode of pancreatitis. Computed tomography of the abdomen showed thick-walled U-shaped cystic structure lying between the head of the pancreas, the inferior gastric antrum and the proximal descending duodenum. An abdominal US demonstrated a bi-lobed thick-walled cystic mass with a positive rim sign. Recurrent pancreatitis is uncommon in pediatric patients. Causes of recurrent pancreatitis in pediatrics are different than those in adults. While hereditary pancreatitis is one of the causes, the genetic testing was negative and there was no sign of pancreatic injury or inflammation in any of the images. Pancreatic pseudocyst is a complication of acute pancreatitis and usually has a thin wall instead of a thick wall, and it's unlikely to be present during the first episode of pancreatitis. Foregut duplication cyst is a rare (about 1 in 4,500) congenital anomaly that can happen anywhere throughout the GI tract. The US finding of a rim sign is a typical appearance of a duplication cyst and usually is managed by surgical resection. Even though foregut duplication is a rare congenital anomaly, it should always be considered in the differential diagnosis of cases of chronic pancreatis, especially with the presence of thick walled cystic formation on imaging studies.
\end{abstract}

Keywords: Foregut duplication; Chronic pancreatits; Pseudocyst

\footnotetext{
Manuscript accepted for publication March 20, 2013

${ }^{\mathrm{a}}$ Mayo Clinic College of Medicine, 200 first street SW, Rochester, MN 55901, USA

${ }^{\mathrm{b} C o l l e g e}$ of Medicine, Division of Pediatric Gastroenterology and Hepatology, 200 first street SW, Rochester, MN 55901, USA

${ }^{\mathrm{c} C}$ Corresponding author: Imad Absah, Mayo Clinic College of Medicine, 200 first street SW, Rochester, MN 55901, USA.

Email: absah.imad@mayo.edu
}

doi: http://dx.doi.org/10.4021/jmc1181w

\section{Introduction}

Duplication of the gastrointestinal tract (GI) tract was first described by Gross in 1952 referring to the extra luminal tract that is contiguous with GI tract and lined by alimentary epithelium and develop a smooth muscle layer [1]. These congenital abnormalities of the GI tract are rare and their incidence is reported to be 1:4,500 [2]. Duplication of the GI tract can happen anywhere from the tongue to the anus. Big case series of 42 cases found that most of these duplication were intraabdominal and cystic in nature [3]. Duplication cysts can be diagnosed at any age, but usually in the first year of life [3]. They become symptomatic either due to ectopic gastric or pancreatic mucosa or by mass affect compressing adjacent structures. There symptoms mostly depends on the location and can include respiratory distress, abdominal pain, bleeding, and pancreatitis. Gastric and duodenal mucosa are rare and account for $5-7 \%$ of the GI tract duplications and tend to be cystic more than tubular [4]. We report a case of antral and duodenal duplication that was thought to be a pancreatic pseudocyst resulting from idiopathic chronic pancreatitis, where it was actually a case of forgot duplication causing the pancreatitis.

\section{Case Report}

A 7-year-old boy presents with a history of recurrent pancreatitis. His complains of abdominal pain started 3 years prior to his first episode of pancreatitis. This abdominal pain was nonspecific and was associated with decrease appetite and sporadic episodes of vomiting. These episodes were intermittent and self resolving. There is no history of abdominal trauma and his initial episode of pancreatitis was evaluated extensively and it was considered idiopathic. Since that time has had at least five known episodes of pancreatitis with mild elevation of his amylase and lipase (range: $200-300$ ), he had negative genetic testing for the hereditary pancreatitis common mutations (CFTR, PRSS1, and SPINK1 gene mutations) [5]. He was found to be heterozygous for HLA DQ2, but celiac serologies were negative. He had numerous imag- 


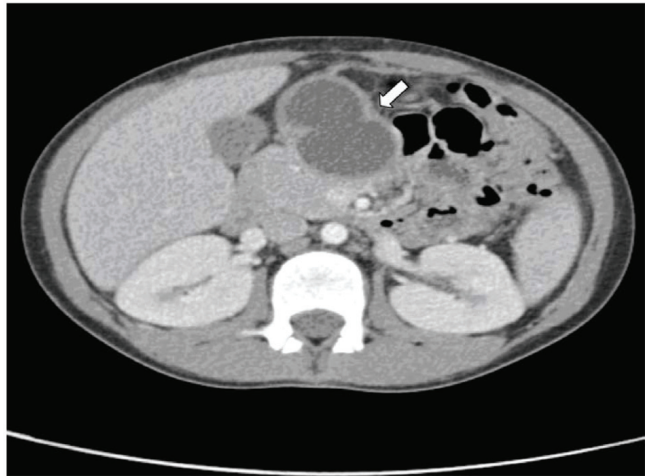

Figure 1. Axial abdominal CT image showing the cystic lesion.

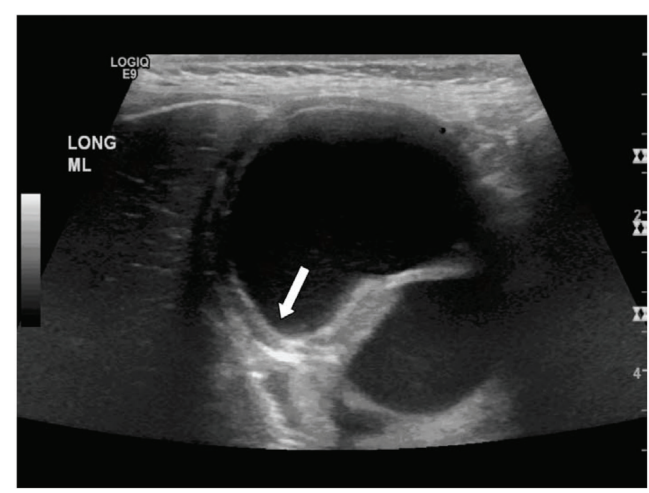

Figure 2. US of the abdomen showing the typical rim sign. ing studies including abdominal ultrasounds (US), computed tomography (CT) scans, and magnetic resonance cholangiopancreatography (MRCP) revealing a persistent cystic lesion near the pancreatic head. This cyst was present since his first episode of pancreatitis and it was always thought to be a pseudocyst that resulted from his initial episode of acute pancreatitis. There was a concern that this pseudocyst is compressing his common pancreatic duct and he was referred to our gastroenterology clinic for ERCP (Endoscopic Retrograde Cholangiopancreatography) and possible drainage and stent placement. After the initial visit the decision was to repeat some of the abdominal images including Abdominal CT and US before doing an ERCP on him. His abdominal CT scan showed a thick-walled U-shaped cystic structure lying between the head of the pancreas, the inferior gastric antrum and the proximal descending duodenum (Fig. 1). The pancreas otherwise was normal in appearance with normal enhancement and no ductal dilatation or calcification. An abdominal US demonstrated a bilobed thick-walled cystic mass with a positive rim sign (Fig. 2). These results were reviewed by our pediatric radiologist and our pediatric surgeon, it was agreed that the presence or rim sign on the US and the thickness of the cystic wall were more consistent with forgot duplication, rather than a pseudocyst. Our patient underwent a laparoscopic abdominal exam that confirmed the diagnosis and Identified a cystic formation of the antrum and first segment of the duodenum representing a forgot duplication. His pancreas had normal appearance the cystic formation was drained and resected. Our patient recovered quickly after the procedure and was discharged home on no medication.

\section{Discussion}

This is a case of recurrent pancreatitis, which is uncommon in pediatric patients. Causes of recurrent pancreatitis in pediatrics are different than those in adults. While hereditary pancreatitis is one of the causes that should be excluded by genetic testing for (CFTR, PRSS1, and SPINK1 gene mutations) [5] and this was negative. Also the imaging studies showed no sign of pancreatic inflammation of duct injury in any of the imaging this patient had. Pancreatic pseudocyst (B) is a complication of acute pancreatitis and usually has a thin wall instead of a thick wall, and it's unlikely to be present during the first episode of pancreatitis. Foregut duplication cyst is a rare (about 1 in 4,500) congenital anomaly that can happen anywhere throughout the GI tract. The US finding of a rim sign is a typical appearance of a duplication cyst (Fig. 2) and usually is managed by surgical resection of the duplication cyst. Intraabdominal malignancies are unusual in this age group. These malignancies are usually are solid with some heterogenic appearance instead of cystic on imaging.

Forgot duplication is rare GI presentation, but should be thought of in cases of recurrent pancreatitis in patients especially when cystic lesions are present near the pancrease.

\section{References}

1. Gross RE, Holcomb GW, Jr., Farber S. Duplications of the alimentary tract. Pediatrics. 1952;9(4):448-468.

2. Schalamon J, Schleef J, Hollwarth ME. Experience with gastro-intestinal duplications in childhood. Langenbecks Arch Surg. 2000;385(6):402-405.

3. Karnak I, Ocal T, Senocak ME, Tanyel FC, Buyukpamukcu N. Alimentary tract duplications in children: report of 26 years' experience. Turk J Pediatr. 2000;42(2):118-125.

4. Macpherson RI. Gastrointestinal tract duplications: clinical, pathologic, etiologic, and radiologic considerations. Radiographics. 1993;13(5):1063-1080.

5. Lucidi V, Alghisi F, Dall'Oglio L, D'Apice MR, Monti L, De Angelis P, Gambardella S, et al. The etiology of acute recurrent pancreatitis in children: a challenge for pediatricians. Pancreas. 2011;40(4):517-521. 